\title{
REM-sleep deprivation and the food-consumption patterns of male rats
}

\author{
RANDALL K. MARTINEZ, JOSE BAUTISTA, NATHAN PHILLIPS, and ROBERT A. HICKS \\ San Jose State University, San Jose, California
}

To clarify ambiguities in earlier studies and to provide a test of the hypothesis that REM-sleep deprivation potentiates primary drive-linked behaviors, the food intake of 15 male rats was monitored continuously for 10 consecutive 24-h periods. Relative to the controls, the REM-deprived animals did not show a significant increase in food intake during the treatment period. However, during this period, these animals showed a pronounced change in their light:dark-period foodintake pattern that persisted into the recovery period. The implications of these data for existing theory and for certain ambiguities in the relevant literature are discussed.

As a partial explanation of the behavioral functions of REM sleep, Vogel (1979) suggested that this stage of sleep plays a role in the modulation of level of generalized drive. He stated that "In animals, RSD (REM-sleep deprivation) increases such drive motivated behaviors as sex, aggression, pleasure seeking, food seeking and grooming." He then added that "a motivational function of RS (REM sleep) is inferred from these observations that RSD increases drive motivated behavior"' (Vogel, 1979, p. 234). That is, this hypothesis stipulates that RSD alters wakeful activity by increasing the level of generalized drive. This inference is partially validated by data from studies that have demonstrated that RSD potentiates primary drive-linked behaviors. In this context, the most extensive set of evidence that is relevant to this hypothesis is a group of several studies designed to measure relationships between REM sleep and the eating behaviors of laboratory animals (e.g., Bergmann, Kushida, Hennessy, Winter, \& Rechtschaffen, 1984; Bowersox, Baker, \& Dement, 1984; Dement, 1965; Elomaa, 1981, 1985; Elomaa \& Johansson, 1980; Siegel, 1975). Essentially, these studies have followed one of two designs: (1) studies using cats, in which eating subsequent to natural fluctuation in REM sleep is monitored or (2) studies using rats, in which eating as a function of RSD is monitored.

\section{Normal Fluctuations in REM Sleep \\ and Food Intake}

In the first study of this type, Siegel (1975) measured the relationships between natural fluctuations in amount of REM sleep and subsequent food consumption in 6 cats. To do this, he continuously monitored certain sleep and food intake parameters of these animals over periods that ranged from 5 to 9 days; for each animal, he computed

\footnotetext{
This research was supported by NIH-MBRS Grant GM 08192-11. Requests for reprints should be addressed to Robert A. Hicks, Department of Psychology, San Jose State University, San Jose, CA 95192.
}

a correlation coefficient between amount of REM sleep and subsequent food intake. Four of the six correlation coefficients were significantly different from zero and the median correlation between these variables for all animals was -.79 . Siegel concluded that "in all but one of the cats, the correlation between REM sleep and subsequent food intake was negative." He then noted that "REM sleep was a better predictor of food intake than either waking, slow wave sleep or previous food intake" (Siegel, 1975, p. 399). However, Bowersox et al. (1984) failed to replicate Siegel's results. That is, while they computed negative correlations between REM sleep and subsequent food intake for 3 of their 5 animals, none of these values were significantly different than zero. They concluded that "this study found no consistent evidence that in the undisturbed animal, food consumption is related quantitatively to daytime/nighttime expression of any sleep or waking variable"' (Bowersox et al., 1984, p. 909). Thus, the relationship between natural fluctuations in REM sleep and subsequent food intake may not be as robust as Siegel's results first indicated.

\section{REM Deprivation and Food Intake}

In the first study of this type, Dement (1965) measured the effect of RSD on the food intake of cats. These data were used as the basis for a presented paper, and the only published record of this work is the following abstract:

Seventeen cats had their food intake carefully measured. By comparison with control cats, there was only some slight trend for the deprived cats to eat more. However, the reverse procedure (i.e., starvation) was attempted, and the motivation to eat was strikingly increased in REM-deprived cats as opposed to controls. (p. 406)

Thus, in this study, Dement noted a slight negative correlation between amount of REM sleep and food intake that was consistent with the results of the Bowersox et al. (1984) study. Perhaps of greater significance, he reported that RSD substantially augmented the effect that food deprivation had on subsequent food intake. 
Elomaa and Johansson (1980) continuously monitored the feeding behaviors of 8 male rats for 8 days. They reported that during the RSD treatment portion of this period, overall, their animals increased food intake by $32 \%$ over baseline, ate more frequently, and exhibited a pronounced reduction in the difference in food intake between the light and dark periods of each 24-h period during the RSD treatment. However, in a subsequent replication of this study, Elomaa (1981) reported that during the RSD treatment, his animals did not increase their food intake significantly, although they did eat more frequently and the normal light-dark difference in meal size was abolished. He explained the difference in the amount of food intake between these studies by asserting that, in the first study, the animals were only partially REMdeprived (he estimated 50\%), whereas, in the second study, his animals were totally deprived of REM sleep. In doing this, Elomaa did not specify the nature of the underlying mechanism(s) that could cause partial RSD and complete RSD to affect food intake differently. Since both of these studies did not include the electrophysiological measurement of sleep, his assumption of a difference in the amount of RSD induced in the animals in these studies was not validated. In summarizing this work, Elomaa (1985) stated that RSD “does not induce true hyperphagia but on the contrary may interfere with the ability of the organism to adapt to increased energy demands"' (p. 26).

The results of a study by Bergmann et al. (1984) further complicates Elomaa's speculation concerning the dynamics of the RSD-food-intake relationship. They deprived rats of REM sleep chronically (RSD was almost total, i.e., $1.3 \%$ of baseline), until these animals died; they reported that, during this period, average food intake increased by $186.2 \%$ over baseline levels. In sharp contrast to Elomaa's assumption, these data suggest that total RSD may potentiate substantial increases in food intake.

The results of the studies considered in this section are in conflict, and, perhaps, this conflict can best be explained by referring to the original study in this group. As was noted, Dement (1965) found that RSD increased food intake in hungry but not in well-fed animals. A common feature of the Elomaa and Johansson (1980) and the Bergmann et al. (1984) studies was that, in both of these, the animals lost a substantial amount of weight during the RSD treatment period, whereas Elomaa (1981) had refined his RSD procedure to eliminate most of this weight loss. The excessive weight loss in the former studies could indicate exposure to a salient stressor and/or be a sign that the ad-lib feeding procedures that were used prevented these animals from obtaining adequate nutrition. Since either of these possibilities confound interpretation of their results, the RSD-food-intake relationships proclaimed by Elomaa and Johansson and Bergmann et al. should be viewed cautiously as the result of the covariation of RSD and food deprivation and/or stress. Thus, the purpose of this study was to measure the relationships between RSD and certain food consumption parameters in a manner that minimized these potential sources of confounding.

\section{METHOD}

\section{Animals}

The animals were 15 male Sprague-Dawley-strain rats that were 46 days old when they were delivered from the supplier (Simonsen Laboratories, Gilroy, CA). After a 14-day adaptation period, the animals were randomly assigned to one of three treatment conditions: the dry control (DC) group, the wet control (WC) group, or the RSD group (there were 5 animals in each group).

\section{REM-Sleep Deprivation}

RSD was achieved using the water-tank procedure, which is described in detail by Hicks and Moore (1979). During the experiment, all of the animals were housed in 18.9-liter buckets, which were modified so that food and water were available ad lib from a feeder on the side of this cage. The top of each bucket was covered with wire mesh; the water bottle was positioned so that the animal could easily reach the spout. During the treatment period, each animal in the RSD condition spent its time in the bucket on a $6.5-\mathrm{cm}$ platform that was surrounded by water $\left(19^{\circ} \mathrm{C}\right)$ to within $1 \mathrm{~cm}$ of the platform. The animals in the WC group were treated identically, with the exception that these animals spent the entire treatment period on a $16.5-\mathrm{cm}$ platform that was surrounded by water. These platforms were large enough to prevent the animals from falling into the water at the onset of REM sleep. Thus, this group served as a non-RSD control for exposure to the wet environment. The DC group spent the entire treatment period housed in a dry RSD apparatus with a $16.5-\mathrm{cm}$ platform in place and therefore served as a non-RSD control for exposure to the novel RSD apparatus.

\section{Monitoring of Food Intake}

The food-intake monitoring system was specially constructed for this study. The feeder was a plastic block, with a $2 \mathrm{~cm}$ diameter $\times 1 \mathrm{~cm}$ deep cone-shaped feeding trough drilled into the top of it, that was mounted into the side of the bucket. Photobeam sensors attached to either side of the feeder trough were plugged into a Colbourn Photobeam Detector (Model 523-01), a Colbourn One-Shot (Model S52-02), and a Colbourn Electronic Counter (Model 523-25). These instruments were wired to a Davis Scientific Instruments Food Dispenser (Model PD104) and interfaced with an Apple Ile computer programmed to keep time, count the number of food pellets dispensed, store data on a floppy disk, and print out data.

When a rat took a food pellet (Noyes, Formula $A, 45 \mathrm{mg}$ ) from the cone-shaped trough, the photobeam would detect it. A signal was then relayed through the Colbourn instruments to the food dispenser and another pellet was released. Thus, there was always a food pellet in the feeder trough available for the animal. This feeding system was developed as the result of considerable pilot effort, and the system that evolved from this work provided easy ad-lib access to food and almost completely eliminated mechanical problems in the delivery of food pellets.

\section{Procedure}

After they were delivered by the supplier, each animal went through a 14-day adaptation period. During this period, and throughout the remainder of the experiment, the animals were housed individually in 18.9liter buckets. During the adaptation period and the recovery period, these buckets were set up to meet the DC condition that is described above. Throughout the adaptation period, the animals were weighed and handled for 5 min each day. After 7 days of this period had elapsed, the food dispenser was introduced, so that each animal could learn to eat from it prior to the start of the treatment period. After the 14-day adaptation period, we recorded food intake continuously for the next 10 days (i.e., for a 2-day baseline period, a 4-day treatment period, and a 4-day recovery period). Monitoring of food intake was started at $1400 \mathrm{~h}$ on the first baseline day and continued until $1400 \mathrm{~h}$ on the last recovery day. Except for the daily $1-\mathrm{h}$ periods required to clean the buckets, add food pellets, weigh the animals, and change the water, there was no disruption of food-intake recording. The computer was programmed to count the number of pellets taken per minute, and it stored the data on a disk every $30 \mathrm{~min}$. The computer would also print out the data every 
$24 \mathrm{~h}$. The feeding apparatus and the computer were checked frequently throughout the day to ensure proper operation. Also, the animals were kept in a red-light condition during the dark cycle to allow for routine nighttime checks.

Throughout the entire experimental period, the lab temperature was $26^{\circ} \pm 1^{\circ} \mathrm{C}$, and the animals were maintained on a 12:12-h light:dark cycle, with lights on at $0800 \mathrm{~h}$.

\section{RESULTS}

First, the body-weight data for each treatment group for each day of the 10-day food-intake measurement period were analyzed by computing a 3 (treatments) $\times 10$ (days) factor analysis of variance, with repeated measures in the second factor (days). This ANOVA was computed to check on the possibility that the treatments had differentially affected stress levels and/or food intake, with the result that the main effect for treatments was not significant $[F(2,12)=1.52]$.

Table 1 summarizes the total food consumption (number of pellets) for each 24 -h period of the 10-day foodintake measurement period for each treatment group.

The food-consumption data were analyzed by computing a 3 (treatments) $\times 10$ (days) factor analysis of variance, with the result that the main effect for treatments was not significant $[F(2,12)=0.67]$. However, both the main effect for days $[F(9,108)=8.36, p<.01]$ and the treatments $\times$ days interaction $[F(18,108)=2.07, p<$ $.05]$ were significant. The significant effect for days probably reflects the general rise in food intake during the treatment period and the decline in food intake during the recovery period; however, the marginally significant interaction is more difficult to explain systematically.

To test Elomaa's (1981) conclusion that RSD abolished light-dark differences in meal size, the differences in total food consumption for each treatment group between each 12-h light and 12-h dark interval for the 10-day foodintake measurement period were evaluated. These data, which are summarized in Table 2, were analyzed by computing a 3 (treatments) $\times 10$ (days) factor analysis of variance. The results of this analysis revealed significant main effects for both treatments $[F(2,12)=4.98, p<.05]$ and days $[F(9,108)=10.57, p<.01]$ and a significant treatment $\times$ days interaction $[F(18,108)=5.29, p<.01]$.

To further elaborate the light-dark food-consumption differences data, separate one-way analyses of variance were computed between treatment groups for each treatment and recovery day. The results of these post hoc analyses suggest significantly smaller light-dark differences

Table 1

The Means and Standard Deviations for the Number of Pellets Consumed by Each Treatment Group on Each Day of the 10-Day Food-Intake Measurement Period

\begin{tabular}{|c|c|c|c|c|c|c|c|c|c|c|}
\hline & \multicolumn{2}{|c|}{ Baseline Days } & \multicolumn{4}{|c|}{ Treatment Days } & \multicolumn{4}{|c|}{ Recovery Days } \\
\hline & 1 & 2 & 3 & 4 & 5 & 6 & 7 & 8 & 9 & 10 \\
\hline \multicolumn{11}{|c|}{ RSD Group } \\
\hline $\begin{array}{l}M \\
S D\end{array}$ & $\begin{array}{r}538.4 \\
63.5\end{array}$ & $\begin{array}{r}521.0 \\
79.5\end{array}$ & $\begin{array}{r}584.2 \\
99.1\end{array}$ & $\begin{array}{r}519.0 \\
94.7\end{array}$ & $\begin{array}{r}603.8 \\
59.5\end{array}$ & $\begin{array}{r}627.4 \\
27.8\end{array}$ & $\begin{array}{r}377.4 \\
48.3\end{array}$ & $\begin{array}{r}449.8 \\
38.8\end{array}$ & $\begin{array}{r}460.8 \\
51.7\end{array}$ & $\begin{array}{r}461.4 \\
45.1\end{array}$ \\
\hline \multicolumn{11}{|c|}{ WC Group } \\
\hline $\begin{array}{l}M \\
S D\end{array}$ & $\begin{array}{r}547.0 \\
63.0\end{array}$ & $\begin{array}{r}528.4 \\
80.2\end{array}$ & $\begin{array}{r}638.8 \\
69.4\end{array}$ & $\begin{array}{r}522.0 \\
29.2\end{array}$ & $\begin{array}{l}586.2 \\
168.0\end{array}$ & $\begin{array}{r}570.0 \\
97.1\end{array}$ & $\begin{array}{r}443.8 \\
61.2\end{array}$ & $\begin{array}{r}503.6 \\
76.0\end{array}$ & $\begin{array}{r}518.4 \\
86.4\end{array}$ & $\begin{array}{r}494.6 \\
76.8\end{array}$ \\
\hline \multicolumn{11}{|c|}{ DC Group } \\
\hline $\begin{array}{l}M \\
S D\end{array}$ & $\begin{array}{r}500.2 \\
56.8\end{array}$ & $\begin{array}{r}552.2 \\
42.0\end{array}$ & $\begin{array}{r}512.2 \\
44.8\end{array}$ & $\begin{array}{r}528.0 \\
43.5\end{array}$ & $\begin{array}{r}512.4 \\
43.2 \\
\end{array}$ & $\begin{array}{r}548.2 \\
76.8 \\
\end{array}$ & $\begin{array}{r}508.6 \\
78.3\end{array}$ & $\begin{array}{r}500.2 \\
55.9\end{array}$ & $\begin{array}{r}504.6 \\
58.1\end{array}$ & $\begin{array}{r}479.6 \\
25.3\end{array}$ \\
\hline
\end{tabular}

Table 2

The Means and Standard Deviations for the Dark:Light Period Differences in Number of Pellets Consumed Each Treatment Day on Each Day of the 10-Day Food-Intake Measurement Period

\begin{tabular}{|c|c|c|c|c|c|c|c|c|c|c|}
\hline & \multicolumn{2}{|c|}{ Baseline Days } & \multicolumn{4}{|c|}{ Treatment Days } & \multicolumn{4}{|c|}{ Recovery Days } \\
\hline & 1 & 2 & 3 & 4 & 5 & 6 & 7 & 8 & 9 & 10 \\
\hline \multicolumn{11}{|c|}{ RSD Group } \\
\hline$M$ & 238.0 & 221.0 & 114.8 & 92.6 & 143.8 & 155.4 & 222.8 & 201.4 & 248.0 & 248.6 \\
\hline$S D$ & 51.4 & 117.0 & 64.9 & 67.5 & 32.2 & 54.4 & 94.9 & 133.0 & 57.7 & 76.8 \\
\hline \multicolumn{11}{|c|}{ WC Group } \\
\hline$M$ & 379.8 & 355.2 & 148.0 & 251.2 & 346.6 & 250.0 & 325.4 & 304.8 & 345.4 & 356.2 \\
\hline$S D$ & 71.6 & 52.0 & 109.0 & 62.2 & 194.0 & 125.0 & 74.0 & 41.8 & 42.6 & 19.3 \\
\hline \multicolumn{11}{|c|}{ DC Group } \\
\hline$M$ & 264.2 & 245.4 & 308.3 & 324.0 & 322.2 & 348.8 & 361.4 & 342.0 & 363.4 & 326.0 \\
\hline$S D$ & 89.2 & 97.3 & 65.4 & 133.0 & 71.2 & 82.8 & 73.3 & 59.8 & 38.8 & 67.4 \\
\hline
\end{tabular}

Note-RSD = REM-sleep deprivation, WC $=$ wet control, and DC = dry control. 
in food consumption for the RSD group throughout the treatment period and on all but the second day of the recovery period. While the data summarized in Table 2 do not indicate that light-dark differences were abolished, as Elomaa (1981) had claimed, these results suggest that REM-deprived rats tend to distribute their food intake more evenly over the light and dark periods and that this pattern of eating persists into the recovery period. Thus, we agree with Elomaa (1985) that RSD does not seem to alter amount of food intake significantly but it does disrupt the normal light-dark eating pattern of these animals.

\section{DISCUSSION}

The analysis of the data summarized in Table 1 indicates that RSD did not significantly increase the food intake of our animals. These data are consistent with the results of an earlier study by Elomaa (1981) but are at odds with the outcome of studies by Elomaa and Johansson (1980) and Bergmann et al. (1984). Perhaps this discrepancy can best be explained by referring to Dement's (1965) research. As was noted, he found that RSD potentiated striking increases in the food intake only when his animals were first deprived of food. In this regard, an important difference between the studies with rats that have and have not concluded that RSD increases food intake is that, in the former, the animals lost considerable weight during the treatment period and, in the latter, significant weight change did not occur as an effect of treatment. While it remains an interesting but unresolved question why RSD seems to interact with food deprivation to potentiate substantial increases in food intake, it seems clear that Elomaa's (1985) conclusion that RSD does not induce true hyperphagia is correct. Thus, these data question Vogel's (1979) assertion that, in general, RSD increases primary drive-motivated behaviors.

The analysis of the data summarized in Table 2 indicates that RSD significantly altered the normal eating patterns of the animals in the RSD group, both during the treatment period and for a period of days during the recovery period. These data are consistent with results of earlier studies (Elomaa, 1981; Elomaa \& Johansson, 1980; Johansson \& Elomaa, 1986) in demonstrating that RSD distributes periods of food intake more evenly over the light:dark cycle than is normally the case. An obvious explanation for the greater light-period food intake in these animals is that REM-deprived rats were awakened more frequently during the light period during treatment and thus were more active than were the control animals during this period. However, this explanation does not account for the fact that the RSD-treated animals continued to show altered patterns of food intake during the posttreatment recovery period; as a consequence (as is shown in Table 2), the light-dark differences in food consumption for the REM-deprived animals remained smaller than did those of the control animals throughout the recovery period. We suspect that these data suggest a carryover effect of a cycle shift that occurred as a function of RSD; however, both the physiological mechanisms and the behavioral parameters of this effect remain to be determined.

\section{REFERENCES}

Bergmann, B., Kushida, C., Hennessy, C. C., Winter, D., \& ReChTSCHAFFen, A. (1984). Paradoxical sleep deprivation in the rat: II. Energy consumption. Sleep Research, 13, 185.

Bowersox, S. S., BAKER, T. L., \& DEMENT, W. C. (1984). Rapid eye movement sleep and its relationship to feeding behavior in the adult cat. Physiology \& Behavior, 32, 378-381.

DEMENT, W. C. (1965). Recent studies on the biological role of REM sleep. American Journal of Psychiatry, 122, 404-408.

ElomaA, E. (1981). The light/dark difference in meal size in the laboratory rat on a standard diet is abolished during REM sleep deprivation. Physiology \& Behavior, 26, 487-493.

ElomaA, E. (1985). Effects of rapid eye movement sleep deprivation on the feeding behavior in the laboratory rat with a description of the cuff pedestal technique. Acta Physiologica Scandinavica, 125(Suppl. 545), 1-35.

ElomaA, E., \& Johansson, G. G. (1980). Rapid eye movement stage of sleep participates in the generation of the nocturnal meal pattern in the rat. Physiology \& Behavior, 24, 331-336.

Hicks, R. A., \& MOORE, J. D. (1979). REM sleep deprivation diminishes fear in rats. Physiology \& Behavior, 22, 689-692.

Johansson, G. G., \& ElomaA, E. (1986). Effects of partial food restriction on nocturnal meal size and feeding speed are counteracted by concurrent REM sleep deprivation in the rat. Behavioral Brain Research, 20, 275-280.

SIEGEL, J. M. (1975). REM sleep predicts subsequent food intake. Physiology \& Behavior, 15, 399-403.

VoGEL, G. W. (1979). A motivational function of REM sleep. In R. Drucker-Colin, M. Shkurovich, \& M. D. Sterman (Eds.), The functions of sleep (pp. 233-250). New York: Academic Press.

(Manuscript received April 1, 1991.) 